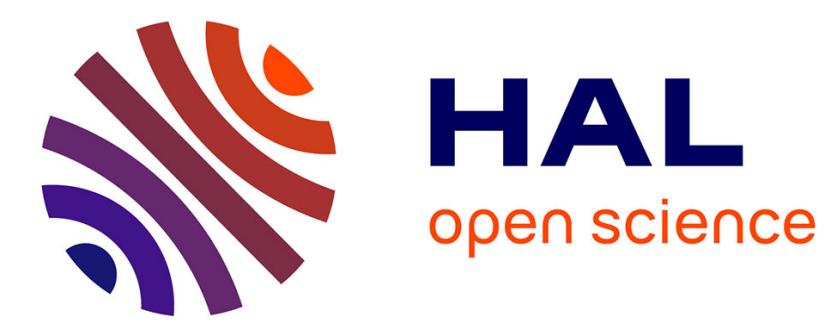

\title{
Resistive transitions in regular superconducting wire networks
}

\author{
S. Teitel, C. Jayaprakash
}

\section{To cite this version:}

S. Teitel, C. Jayaprakash. Resistive transitions in regular superconducting wire networks. Journal de Physique Lettres, 1985, 46 (1), pp.33-38. 10.1051/jphyslet:0198500460103300 jpa-00232473

\section{HAL Id: jpa-00232473 https://hal.science/jpa-00232473}

Submitted on 1 Jan 1985

HAL is a multi-disciplinary open access archive for the deposit and dissemination of scientific research documents, whether they are published or not. The documents may come from teaching and research institutions in France or abroad, or from public or private research centers.
L'archive ouverte pluridisciplinaire HAL, est destinée au dépôt et à la diffusion de documents scientifiques de niveau recherche, publiés ou non, émanant des établissements d'enseignement et de recherche français ou étrangers, des laboratoires publics ou privés. 
Classification

Physics Abstracts

$74.10-74.70-64.90$

\title{
Resistive transitions in regular superconducting wire networks
}

\author{
S. Teitel \\ Department of Physics, The Weizmann Institute of Science, Rehovot 76100, Israel \\ and C. Jayaprakash \\ Department of Physics, The Ohio State University, Columbus, OH 43210, U.S.A.
}

(Reçu le 7 août 1984, accepté le 6 novembre 1984)

\begin{abstract}
Résumé. - On considère la transition résistive d'une grille bidimensionnelle infinie et régulière de fils supraconducteurs. On montre que la transition n'est pas déterminée avec précision par les théories de Landau-Ginzburg linéarisées : ceci est dû aux fluctuations de type vortex. En supposant l'amplitude constante, on construit un modèle non linéaire dont les riches propriétés, sous la température de transition (en champ moyen) sont très similaires à celles prédites pour les réseaux de jonctions Josephson. On compare les résultats à des expériences récentes.
\end{abstract}

\begin{abstract}
The resistive transition of a two dimensional infinite regular grid of superconducting wires is considered. Previous linearized Landau-Ginzburg theories of this transition are shown to be inadequate in determining the true phase boundary due to the effects of vortex fluctuations. The relation of the non linear model to a constant amplitude approximation is discussed and rich structure, identical to that previously predicted for Josephson junction arrays, is expected below the mean field transition temperature. Connection with recent experiments is made.
\end{abstract}

Within the last two years, extensive theoretical and experimental effort has been devoted to the problem of two dimensional superconducting networks in a magnetic field [1-15]. Although much of this interest is concerned with the problem of disordered systems at the percolation threshold [4-11], considerable theoretical attention has been given to the problem of an infinite regular wire grid [5-7a, 12], and interesting properties as a function of applied field $H$ have been predicted. Recent experiments by Pannetier et al. [13] on such a regular system provide further stimulation to renew study of this problem.

Previously, most analyses of this problem have centred on the solution of the linearized Landau-Ginzburg equations for the superconducting wave function on the wire links $[5,7 \mathrm{a}, 12]$. Although such a mean field like treatment may be expected to determine the temperature at which a decrease from the normal state resistance begins, the absence of long range order in two dimensions means fluctuation effects must be included. We expect, as has been predicted and observed in other 2D superconducting systems [16], that below the mean field transition temperature there remains a flux flow resistive tail due to field induced and thermally excited vortex fluctuations. In this paper we derive the qualitative behaviour of the phase boundary of the 
true resistive transition where these resistive tails vanish. A general fluctuating non linear LandauGinzburg model is considered and found to yield rich behaviour equivalent to that previously predicted for Josephson junction arrays [14, 15]. Considerations relevant for experimental observation of these effects are discussed.

Let $\left\{\psi_{i}\right\}$ be the values of the superconducting wave function at the nodes $\{i\}$ of an infinite two dimensional regular square grid of wires. As usual [17] we ignore inductive couplings between wire loops and take $\mathbf{A}$ as the vector potential of a uniform applied magnetic field $H$ perpendicular to the grid. Since the behaviour of $\psi$ along the link connecting nodes $i$ and $j$ is determined by the values at the endpoints, one can write an effective Hamiltonian $\mathscr{H}$ for the system just in terms of nearest neighbour interactions of the $\left\{\psi_{i}\right\}$. Gauge invariance then requires that $\mathscr{H}\left[\psi_{i}\right]$ have the form

$$
\begin{aligned}
\mathscr{H}\left[\psi_{i}\right]=\sum_{n=1}^{\infty}\left\{a_{n} \sum_{\langle i j\rangle}\left[\psi_{i}^{*} \mathrm{e}^{i A_{i j}} \psi_{j}\right]^{n}+b_{n} \sum_{i}\left|\psi_{i}\right|^{2 n}\right\} & + \\
& +\sum_{n m l=1}^{\infty} c_{n m l} \sum_{\langle i j\rangle}\left[\psi_{i}^{*} \mathrm{e}^{i A_{i j}} \psi_{j}\right]^{n}\left|\psi_{i}\right|^{2 m}\left|\psi_{j}\right|^{2 l}
\end{aligned}
$$

where the sums are over all nodes $i$ and nearest neighbour links $\langle i j\rangle$ and $A_{i j}=(2 e / \hbar c) \int_{i}^{j}$ A.dl. In the following magnetic field $H$ will be measured in units of $f=a^{2} H / \Phi_{0}$, the number of flux quanta of external field per unit cell of the lattice (lattice spacing is $a$ ). For rational flux we will write $f=p / q$ where $p$ and $q$ are coprime.

It is natural to consider two separate approximations to the Hamiltonian (1); the Gaussian, and that of constant amplitude. In the Gaussian approximation one sets all coefficients in (1) to zero except $a_{1}$ and $b_{1}$, and expands $b_{1} / a_{1}$ as a power series in $T_{\text {co }}-T$, where $T_{\text {co }}$ is the mean field $f=0$ transition temperature. The transition temperature for finite $f$ is then determined when the lowest eigenvalue of the resulting quadratic form becomes zero, and the corresponding eigenvector determines the condensed state. This eigenvalue problem, with the particular choice $-b_{1} / a_{1}=z \cos a / \xi_{\mathrm{s}}$ (where $z$ is the coordination member of the lattice, $a$ the lattice constant, and $\xi_{\mathrm{s}} \propto\left(T_{\mathrm{co}}-T\right)^{1 / 2}$ the mean field correlation length) is identical to solving the linearized Landau-Ginzburg equations on the net, which, as pointed out by Alexander [5], is equivalent to the problem of non interacting tight binding electrons in a magnetic field first studied by Azbel [18] and later by Hofstadter [19]. The reduction in $T_{\mathrm{c}}$ for finite $f$ is proportional to the increase in the lowest band edge of this electronic problem. The resulting mean field phase boundary, as obtained from Hofstadter's work, is shown in figure 1a. Although the boundary is very singular, having cusps at all rational $f, T_{\mathrm{c}}(f)$ is still finite and continuous everywhere. Similar results have been obtained from a molecular field analysis of a constant amplitude approximation to (1) by Shih and Stroud [20]. We note that the mean field transition temperatures are such that the inequality $a / \xi_{\mathrm{s}}<1$ is satisfied along the phase boundary of figure $1 \mathrm{a}$. We return to this point when considering the importance of vortex fluctuations.

The constant amplitude approximation is obtained from (1) by writing $\psi_{i}=g_{i} \mathrm{e}^{i \theta_{i}}$ and fixing all amplitudes $g_{i}=1$. The resulting Hamiltonian has the form

$$
\mathscr{H}\left[\theta_{i}\right]=\sum_{\langle i j\rangle} V\left(\theta_{i}-\theta_{j}-A_{i j}\right)+\text { constant }
$$

where $V(\theta)$ is some potential periodic in $2 \pi$. This model belongs to the class of uniformly frustrated $X Y$ models studied in references [14] and [15]. The particular choice of all $a_{n}, b_{n}, c_{n m l}=0$ except $a_{1} \neq 0$ is precisely the Josephson junction array problem. In reference [15] a combination of analytic bounds, Monte Carlo calculations, and physical arguments was used to show that 


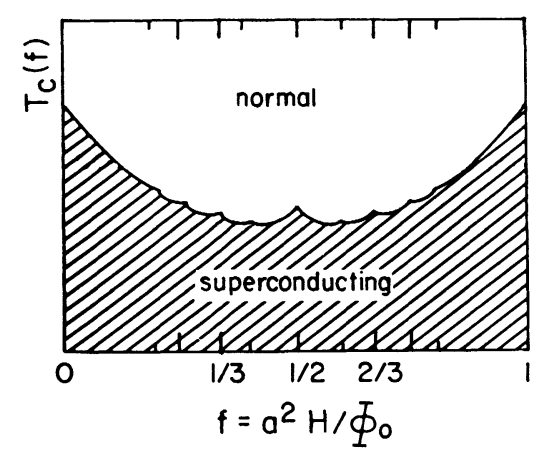

Fig. 1a. - Phase boundary for the resistive transition of a 2D square wire grid in the Gaussian (mean field) model as a function of applied magnetic field $f=a^{2} H / \Phi_{0}$. The boundary is periodically extended on the integers $f$.

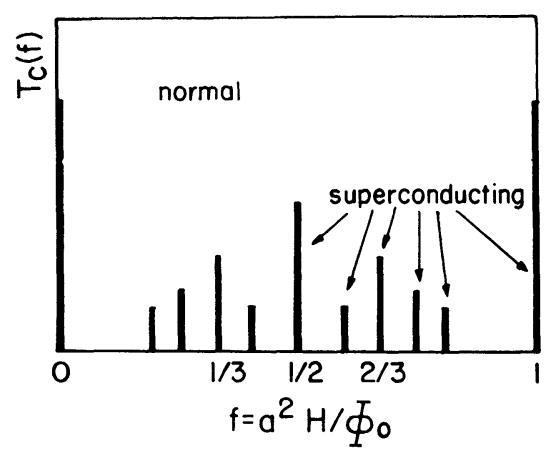

Fig. 1b. - Schematic phase boundary for the resistive transition of a 2D square wire grid in the general non linear model (1) as a function of applied magnetic field $f=a^{2} H / \Phi_{0}$. The boundary consists of infinitely sharp spikes at rational $f=p / q$ with heights bounded by $T_{\mathrm{c}}(p / q) \lesssim$ const. $/ q$, and is periodically extended on the integers $f$.

for the Josephson array with magnetic field $f=p / q\left(f=a^{2} H / \Phi_{0}\right)$ the resistive transition is bounded by

$$
k_{\mathrm{B}} T_{\mathrm{c}}(p / q) \leqslant \pi\left|E_{0}(f)\right| / 2 q
$$

where $E_{0}$ is the ground state energy. The resulting discontinuous phase boundary (due to the $1 / q$ dependence), shown schematically in figure $1 \mathrm{~b}$, consists of a set of infinitely sharp spikes with heights bounded by (3) and is dramatically different from the Gaussian prediction (Fig. 1a).

We now show that the same arguments that applied for the Josephson array continue to hold for the general Hamiltonian (1) with amplitude fluctuations. Consider the behaviour of the critical current of the system. The outline of the argument is then as follows :

i) To drive a supercurrent through a lattice of length $N$, apply twisted boundary conditions to the phase of $\psi$

$$
\psi(N, y)=\psi(0, y) \mathrm{e}^{i N \delta}
$$


For total free energy density $F(\delta)$, the supercurrent induced by this twist is

$$
\frac{\hbar}{2 e} i(\delta, f)=\frac{\partial F}{\partial \delta} \text {. }
$$

The zero temperature critical current is then

$$
i_{\mathrm{c}}(f)=\lim _{T \rightarrow 0} \max _{\delta}[i(\delta, f)] .
$$

ii) Transform to untwisted variables which obey periodic boundary conditions

$$
\chi_{i} \equiv g_{i} \mathrm{e}^{i \phi_{i}}=\psi_{i} \mathrm{e}^{-i x_{i} \delta} .
$$

In terms of $\left\{\chi_{i}\right\}$ the Hamiltonian (1) has the form

$$
\mathscr{H}_{\delta}\left[\chi_{i}\right]=\sum_{\langle i j\rangle} \sum_{n m l=0}^{\infty} \tilde{c}_{n m l} g_{i}^{n+2 m} g_{j}^{n+2 l} \mathrm{e}^{-i n\left(\phi_{i}-\phi_{j}-A_{i j}+\delta \hat{\mathbf{e}}_{i j} \cdot \hat{\mathbf{x}}\right)}+\sum_{n, i} b_{n} g_{i}^{2 n}
$$

where $\hat{\mathbf{e}}_{i j}$ is the unit vector from $i$ to $j$.

iii) For the gauge $\mathbf{A}=y H \hat{\mathbf{x}}$ and $f=p / q, H_{\delta}$ is invariant under $\delta \rightarrow \delta+2 \pi / q$ combined with a translation of the lattice in the $\hat{\mathbf{y}}$ direction $(x, y) \rightarrow(x, y-n a)$, where $n$ is determined by $n p+m q=1(n, m, p, q$ integer). Thus the current $i(\delta, p / q)$ is periodic in $\delta \rightarrow \delta+2 \pi / q$.

iv) Differentiating (5) yields

$$
\frac{\hbar}{2 e} \frac{\mathrm{d} i}{\mathrm{~d} \delta} \leqslant\left\langle\frac{\partial^{2} u}{\partial \delta^{2}}\right\rangle
$$

where $u$ is the energy density. For physically reasonable $\mathcal{H}$, the right hand side of (9) should be bounded as a function of $\delta$ and $f$.

v) Combine the bound (9) with the periodicity (iii) of $i(\delta)$ and the mean value theorem to get a bound on the critical current (6).

$$
\frac{\hbar}{2 e} i_{\mathrm{c}}(p / q) \leqslant\left[\left\langle\frac{\partial^{2} u}{\partial \delta^{2}}\right\rangle\right]_{\max } \frac{\pi}{q} .
$$

vi) Physical arguments then suggest the conclusion

$$
k_{\mathrm{B}} T_{\mathrm{c}}(p / q) \lesssim \frac{\hbar}{2 e} i_{\mathrm{c}}(p / q)
$$

i.e. once thermal noise currents equal the zero temperature $i_{\mathrm{c}}$, a resistive transition should have occurred. ( $i_{\mathrm{c}}$ may be thought of as an effective $T=0$ interface energy between degenerate ground states corresponding to different overall phase factor.)

Combining equations (10) and (11) thus suggests that all Hamiltonians (1) with general amplitude fluctuations should have qualitatively the same behaviour and phase boundary (Eq. (3) and Fig. 1b) as the Josephson array. That the Gaussian model in particular obeys the bound on critical current (10) can be obtained directly from Hofstadter's [19] work by noting that the width of the lowest band obeys a similar relation [21]. The Gaussian model, however, fails to obey the assumption (11) regarding $T_{\mathrm{c}}$ (Fig. 1a). Thus within the Gaussian model one is forced to the unphysical conclusion that for $f$ irrational $(q \rightarrow \infty), T_{\mathrm{c}}(f)$ is finite, but $i_{\mathrm{c}}(f)=0$ for all temperatures. This contradiction is a result of the mean field nature of the approximation, which always gives long range order at a finite critical temperature $T_{\mathrm{c}}$; fluctuation effects, as measured by $i_{\mathrm{c}}$ are ignored. For the constant amplitude approximation of the Josephson array however, 
Monte Carlo simulations [15] at several $f$ supported the assumption (11). For the general Hamiltonian (1), the presence of higher order terms that tend to suppress amplitude fluctuations should make the model approach the constant amplitude approximation and restore the physically reasonable assumption (11). This viewpoint is supported by noting that for $f=0$ (the ordinary 2D $X Y$ model) it has been shown [22] that amplitude fluctuations are irrelevant in a renormalization group sense.

The results of the Gaussian (mean field) model (Fig. 1a) should still remain meaningful as being the approximate temperature where resistance rises to saturate to its high $T$ limit. However, the phase boundary of the true resistive transition, where a non zero linear d.c. resistance first appears, should be as in figure $1 \mathrm{~b}$. The implications for the resulting network resistance have been fully discussed in reference [15]. The arguments presented above are completely generalizable to three dimensions in the case where the field is aligned parallel to one of the grid axes. This conclusion is supported by recent 3D Monte Carlo study by Shih et al. [23].

Physically, the origin of the phase diagram $1 \mathrm{~b}$, is linked to the vortex lattice of circulating currents induced by a non zero $H[14,15]$. The commensurability of this vortex lattice with the underlying periodicity of the wire net, as measured by $q$ where $p / q=H a^{2} / \Phi_{0}$, determines the relative strength of the effective pinning of the vortices. As $T$ increases, the vortices unpin and their motion gives rise to flux flow resistance. This points to an important bound required to see the effects discussed above. When a temperature $T^{*}$ is reached such that $a / \xi_{\mathrm{s}}\left(T^{*}\right) \approx 1$ (where $\xi_{\mathrm{s}}$ is the mean field correlation length) then the effective core of a vortex exceeds the size of a unit cell [6] and the vortex should be regarded as unpinned. $T^{*}$ thus serves as an additional upper bound on the true resistive transition at finite $H$, which in some cases might preempt the commensurability effects discussed above. It is thus necessary to look below $T^{*}$, and hence below the mean field transition temperatures, to see these effects. A second important requirement for observation of commensurability effects is that irregularities in the lattice be small. Impurity pinning due to irregularities should widen the infinitely sharp spikes of figure $1 \mathrm{~b}$ into bands of finite width and wash out some of the fine structure of the phase diagram. We have no way at present to estimate the strength of pinning due to lattice irregularities.

Finally we make two observations regarding the recent experiments on a uniform $2 \mathrm{D}$ honeycomb grid of wires which have been performed by Pannetier et al. [13]. Firstly, we note that for a graph of $R(H)$ at one fixed $T>T_{\text {co }}$ they observe sharp minima at integer $f$ and minima at $f=1 / 3,2 / 3\left(f=a^{2} H / \Phi_{0}\right)$. The appearance of minima at non integer $f$ is consistent with the behaviour predicted above. However, the absence of a minimum at $f=1 / 2$ is in contrast to what has been observed experimentally in square Josephson arrays [24]. Pannetier et al. explain this absence by performing a Gaussian analysis of the honeycomb lattice and noting that the cusp in $T_{\mathrm{c}}(f)$ at $f=1 / 3$ is more pronounced than at $f=1 / 2$ (in contrast to the case of a square lattice, Fig. 1a). Their data appears to be in the mean field range and hence their analysis consistent. However, on the basis of a constant amplitude approximation, a stronger statement can be made. For $f=1 / 2$ and constant amplitude, the problem is that of the fully frustrated $2 \mathrm{D} X Y$ model considered in reference [14]. The ground state maps onto a neutral configuration of $+1 / 2,-1 / 2$ Coulomb charges on the dual lattice. For a square lattice the ground state consists of an ordered lattice of alternating $+1 / 2,-1 / 2$ charges, is doubly degenerate, and was found to melt at a relatively high $T_{\mathrm{c}}$ with Ising exponents [14]. For a honeycomb wire grid however, the charges sit on the dual triangular lattice. The analogy with an Ising antiferromagnet $(+1 / 2=\uparrow,-1 / 2=\downarrow)$ found for the square lattice, applied now to the triangular case [25] suggests a low melting temperature for the ground state vortex lattice. Thus for the honeycomb wire grid, no minimum at $f=1 / 2$ should be expected, even in the resistive tails, below the mean field transition. Secondly, we note that they estimate $a / \xi_{s}(T=0) \approx 10$ while for the lowest value of the mean field transition temperature (at $f=1 / 2) a / \xi_{\mathrm{s}} \simeq 0.6$. It should therefore be possible to conduct measurements in the range $a / \xi_{\mathrm{s}}>1$, and thus if their system is sufficiently pure, it may be suitable to investigate the effects predicted above. 


\section{Acknowledgments.}

It is a pleasure to thank S. Alexander, D. Mukamel, B. Pannetier, D. Stroud and S. Solla for useful discussions. This work was supported in part by NSF grant No. DMR 81-14842, and a Bantrell postdoctoral fellowship. One of us (C. J.) is grateful to the A. P. Sloan Foundation for its fellowship.

\section{Note added :}

It has been pointed out to us by a referee that the sheet resistance in the Pannetier et al. experiment [13] is sufficiently low that according to the results of Beasley et al.(Phys. Rev. Lett.42 (1979) 1165 ) one expects $T_{\mathrm{KT}} \simeq T_{\mathrm{co}}$ and so for $H=0$ only mean field results should be expected. It is not clear to us however that this remains the relevant criteria for observation of non mean field behaviour at finite $H$.

\section{References}

[1] De Gennes, P. G., C. R. Hebd. Sean. Acad. Sci., Ser. B 292 (1981) 9; ibid. 292 (1981) 279.

[2] Fink, H. J., Lopez, A. and Maynard, R., Phys. Rev. B 26 (1982) 5237.

[3] Riess, J., J. Physique Lett. 43 (1982) L-277.

[4] Deutscher, G., Grave, I. and Alexander, S., Phys. Rev. Lett. 48 (1982) 1497.

[5] Alexander, S., Phys. Rev. B 27 (1983) 1541.

[6] Alexander, S. and Halevi, E., J. Physique 44 (1983) 805.

[7] Rammal, R., Lubensky, T. C. and Toulouse, G. (a) Phys. Rev. B 27 (1983) 2820 and (b) J. Physique Lett. 44 (1983) L-65.

[8] Rammal, R. and Toulouse, G., Phys. Rev. Lett. 49 (1982) 1194.

[9] Stephen, M. J., Phys. Lett. A 87 (1981) 67.

[10] Rammâl, R. and Angles d’Auriac, J. C., J. Phys. C 16 (1983) 3933.

[11] Bowman, D. R. and Stroud, D., Phys. Rev. Lett. 52 (1984) 299.

[12] Simonin, J., Rodrigues, D. and Lopez, A., Phys. Rev. Lett. 49 (1982) 944.

[13] Pannetier, B., Chaussy, J. and Rammal, R., J. Physique Lett. 44 (1983) L-853.

[14] Teitel, S. and Jayaprakash, C., Phys. Rev. B 27 (1983) 598.

[15] Teitel, S. and Jayaprakash, C., Phys. Rev. Lett. 51 (1983) 1999.

[16] Cf. Minnhagen, P., in Proceedings of NATO ASI on Percolation, Localization and Superconductivity (1983), to be published, and references therein.

[17] See discussion in Refs. [6] or [15] for example.

[18] Azbel, M. Ya., Sov. Phys. - JETP 19 (1964) 634.

[19] Hofstadter, D. R., Phys. Rev. B 14 (1976) 2239.

[20] Shit, W. Y. and Stroud, D., Phys. Rev. B 28 (1983) 6575.

[21] Kонmoto, M., Kadanoff, L. P. and TANG, C., Phys. Rev. Lett. 50 (1983) 1870.

[22] Nelson, D. R. and Pelcovits, R. A., Phys. Rev. B 16 (1977) 2191.

[23] Shin, W. Y., EBNer, C. and STroud, D., Ohio State University preprint.

[24] Voss, R. F. and Webi, R. A., Phys. Rev. B 25 (1982) 3446;

Webb, R. A., Voss, R. F., Grinstein, G. and Horn, P. M., Phys. Rev. Lett. 51 (1983) 690 ;

Tinkham, M., Abraham, D. W. and Lobb, C. J., Phys. Rev. B 28 (1983) 6578 ;

Kimhi, D., Leyvraz, F. and Ariosa, D., Phys. Rev. B 29 (1984) 1487.

[25] For short range Ising antiferromagnet on a triangular lattice one has the exact result $T_{\mathrm{c}}=0$ (HouTAPPEL, R. M. F., Physica 16 (1950) 426). In the fully frustrated $X Y$ model, the " spins " have long range Coulomb interactions, however we expect the charge neutrality condition to effectively convert these to short range. This is consistent with the exponents found for other lattice geometries see Ref. [14] and also Lee, D. H., Joanopolis, J. D., Negele, J. W. and Landau, D. P., Phys. Rev. Lett. 52 (1984) 433 . Thus we expect a very small $T_{c}$, although perhaps not zero. 